\title{
Thermopower of few-electron quantum dots with Kondo correlations
}

\author{
LvZhou Ye, ${ }^{1}$ Dong Hou,,${ }^{1,2}$ Rulin Wang, ${ }^{1}$ Dewen Cao, ${ }^{1}$ Xiao Zheng,,${ }^{1,2, *}$ and YiJing Yan ${ }^{1,3, \dagger}$ \\ ${ }^{1}$ Hefei National Laboratory for Physical Sciences at the Microscale, University of Science and Technology of China, Hefei, \\ Anhui 230026, China \\ ${ }^{2}$ Synergetic Innovation Center of Quantum Information and Quantum Physics, University of Science and Technology of China, \\ Hefei, Anhui 230026, China \\ ${ }^{3}$ Department of Chemistry, Hong Kong University of Science and Technology, Hong Kong, China \\ (Received 15 June 2013; revised manuscript received 16 September 2014; published 14 October 2014)
}

\begin{abstract}
The thermopower of few-electron quantum dots is crucially influenced by on-dot electron-electron interactions, particularly in the presence of Kondo correlations. In this paper, we present a comprehensive picture which elucidates the underlying relations between the thermopower and the spectral density function of two-level quantum dots. The effects of various electronic states, including the Kondo states originating from both spin and orbital degrees of freedom, are clearly unraveled. Such a physical picture is affirmed by accurate numerical data obtained with a hierarchical equations of motion approach. Our findings and understandings provide an effective and viable way to control the thermoelectric properties of strongly correlated quantum dot systems.
\end{abstract}

DOI: 10.1103/PhysRevB.90.165116

PACS number(s): 72.20.Pa, 71.27.+a, 73.63.Kv, 79.10.-n

\section{INTRODUCTION}

Thermopower is one of the fundamental thermoelectric properties. It measures the thermovoltage $V_{T}$ induced by a temperature gradient $\Delta T$. Materials with a large thermopower are potentially useful for a variety of applications, such as electronic refrigeration [1], thermoelectric conversion [2], and on-chip cooling [3]. The thermopower of nanostructured materials, such as quantum wires, quantum dots (QDs), and molecular junctions, is found to be significantly larger than the prediction of the Wiedemann-Franz law [4,5]. Experimental [5-18] and theoretical works [19-30] on this subject have been extensive.

It has been found that the phonon contribution to the thermopower of QDs is greatly suppressed [31]. Therefore, thermopower can be deemed an intrinsic electronic property, which is very sensitive to the details of electronic structure. The thermopower of a few-electron QD is tunable by varying the discrete energy levels with a gate voltage, as realized by Scheibner et al. on a QD of 20 to 40 electrons [9]. They have observed that the thermopower in the Kondo regime differs distinctly from that in the Coulomb blockade regime [9]. However, the predominant effect leading to such a distinction is rather unclear. Although it would "be interesting to look for these effects in QDs in the very-few-electron limit" [10,32], related theoretical studies have remained scarce.

Costi et al. have explored the spin-Kondo (S-Kondo) effects on the thermopower of a single-level QD [22]. The physical origin of S-Kondo phenomena is the screening of localized electronic spin by the spins of conduction electrons in the surrounding environment. Moreover, a real QD usually consists of more than one level. The strong Coulomb interactions between two degenerate or nearly degenerate levels will give rise to the orbital-Kondo (O-Kondo) phenomena [33,34]. The presence of O-Kondo may substantially enhance the Kondo temperature of a multilevel QD [35]. Sakano et al. have

\footnotetext{
*xz58@ustc.edu.cn

†yyan@ust.hk
}

studied the O-Kondo effects on the thermopower of multilevel QDs [36,37].

Despite the reported progress, a clear and comprehensive physical picture which unravels the complex effects of the various electronic states in a few-electron multilevel QD is still lacking. The main goal of this paper is to provide such a picture and to devise viable means of controlling the thermopower of strongly correlated QDs.

The major challenge for theoretical studies is accurate characterization of Kondo correlations. A number of numerical approaches have been employed to study strongly correlated QDs. These include the numerical renormalization group approach [38-41], the quantum Monte Carlo approach [42,43], the exact diagonalization approach $[44,45]$, the noncrossing approximation [46,47], and the hierarchical equations of motion (HEOM) approach [48-54]. In this paper, we employ the HEOM approach to investigate the thermoelectric properties of single- and two-level QDs.

The remainder of this paper is organized as follows. In Sec. II we introduce the multilevel Anderson impurity model adopted in this work and briefly discuss the basic features of the HEOM approach. In Sec. III we elucidate the underlying relations between the thermopower and the characteristic spectral features of QDs. Our understandings and predictions are then verified with the accurate HEOM numerical results. Finally, some concluding remarks are given in Sec. IV.

\section{MODEL AND METHODOLOGY}

\section{A. Multilevel Anderson impurity model}

We adopt the Anderson impurity model to represent the few-electron QDs of interest in this work. The total Hamiltonian is

$$
H_{\text {total }}=H_{\text {dot }}+H_{\text {lead }}+H_{\text {coup }} \text {. }
$$

The dot is described by

$$
H_{\mathrm{dot}}=\sum_{i} \epsilon_{i} \hat{n}_{i}+U \sum_{i} \hat{n}_{i \uparrow} \hat{n}_{i \downarrow}+U \sum_{i>j} \hat{n}_{i} \hat{n}_{j} .
$$


Here, $\hat{n}_{i}=\sum_{s} \hat{n}_{i s} \equiv \sum_{s} \hat{a}_{i s}^{\dagger} \hat{a}_{i s}$ is the occupation number operator for the $i$ th level, and $\hat{a}_{i s}^{\dagger}\left(\hat{a}_{i s}\right)$ creates (annihilates) an electron of spin $s$ on the $i$ th level of energy $\epsilon_{i}$. The Coulomb repulsion energy $U$ assumes the same value for intralevel and interlevel interactions. The interlevel spacing $\delta \epsilon=\epsilon_{i+1}-\epsilon_{i}$ is usually an order of magnitude smaller than $U$ [55]. The noninteracting electrons in the two leads are described by the Hamiltonian

$$
H_{\text {lead }}=\sum_{\alpha k s} \epsilon_{\alpha k} \hat{d}_{\alpha k s}^{\dagger} \hat{d}_{\alpha k s},
$$

where $\hat{d}_{\alpha k s}^{\dagger}\left(\hat{d}_{\alpha k s}\right)$ creates (annihilates) an electron on the $k$ th state of lead $\alpha$. The interactions between the QD and the leads are described by the Hamiltonian

$$
H_{\text {coup }}=\sum_{\alpha i k s} t_{\alpha i k} \hat{a}_{i s}^{\dagger} \hat{d}_{\alpha k s}+\text { H.c., }
$$

where $\left\{t_{\alpha i k}\right\}$ are the coupling constants.

In the HEOM approach, the influence of leads on the quantum impurities is characterized by hybridization functions, which assume a Lorentzian form of

$$
\begin{aligned}
\Delta_{\alpha, i j}(\omega) & \equiv \pi \sum_{k} t_{\alpha i k} t_{\alpha j k}^{*} \delta\left(\omega-\epsilon_{\alpha k}\right) \\
& =\delta_{i j} \frac{\Delta_{\alpha}}{\left(\omega-\mu_{\alpha}\right)^{2} / W^{2}+1} .
\end{aligned}
$$

Here, $W$ is the band width, and $\mu_{\alpha}$ is the chemical potential of the $\alpha$ th lead. $\Delta=\sum_{\alpha} \Delta_{\alpha}$ is the overall dot-lead coupling strength and is taken to be the energy unit hereafter.

\section{B. The HEOM approach for strongly correlated quantum impurity systems}

The HEOM approach treats quantum impurity systems from the perspective of open dissipative dynamics. The HEOM theory can be established based on a formally exact Feynman-Vernon path-integral formalism [56], as long as the bath environment satisfies Gaussian statistics, which is true for noninteracting electron reservoirs.

The recently developed HEOM approach provides a useful tool for the accurate and efficient characterization of general open quantum systems. It has been employed to investigate a wide range of equilibrium and nonequilibrium and static and dynamic properties of strongly correlated quantum impurity systems $[49,52,53,57,58]$. The numerical results of the HEOM approach are considered to be quantitatively accurate, as long as the results converge with respect to the truncation of the hierarchy.

The derivation of the HEOM formalism for a fermionic environment has been detailed in Refs. [48,50,54,59]. Here, we only introduce some of its basic features. The final form of HEOM can be cast into a compact form as follows [48]:

$$
\begin{aligned}
\dot{\rho}_{j_{1} \cdots j_{n}}^{(n)}= & -\left(i \mathcal{L}+\sum_{r=1}^{n} \gamma_{j_{r}}\right) \rho_{j_{1} \cdots j_{n}}^{(n)}-i \sum_{j} \mathcal{A}_{j} \rho_{j_{1} \cdots j_{n} j}^{(n+1)} \\
& -i \sum_{r=1}^{n}(-)^{n-r} \mathcal{C}_{j_{r}} \rho_{j_{1} \cdots j_{r-1} j_{r+1} \cdots j_{n}}^{(n-1)} .
\end{aligned}
$$

Here, $\rho^{(0)}(t)=\rho(t) \equiv \operatorname{tr}_{\text {env }} \rho_{\text {total }}(t)$ is the reduced density matrix, and $\left\{\rho_{j_{1} \cdots j_{n}}^{(n)}(t) ; n=1, \ldots, L\right\}$ are the auxiliary density matrices, with $L$ being the truncation level.

In Eq. (6) the multicomponent index $j \equiv(\sigma \alpha \mu \nu m)(\sigma=$ $+/-)$ characterizes the transfer of an electron from (to) the impurity level $\mu$ to (from) level $v$ via the $\alpha$ th lead and is associated with a memory time $\gamma_{m}^{-1}$. The Grassmann superoperators $\mathcal{A}_{\bar{j}} \equiv \mathcal{A}_{\mu}^{\bar{\sigma}}$ and $\mathcal{C}_{j} \equiv \mathcal{C}_{\mu \nu m}^{\sigma}$ are defined via their fermionic/bosonic actions on an operator $\hat{O}$ as $\mathcal{A}_{\mu}^{\bar{\sigma}} \hat{O} \equiv\left[\hat{a}_{\mu}^{\bar{\sigma}}, \hat{O}\right]_{\mp}$ and $\mathcal{C}_{\mu \nu m}^{\sigma} \hat{O} \equiv \eta_{\mu \nu m}^{\sigma} \hat{a}_{v}^{\sigma} \hat{O} \pm\left(\eta_{\mu \nu m}^{\bar{\sigma}}\right)^{*} \hat{O} \hat{a}_{\nu}^{\sigma}$, respectively, with $\bar{\sigma}$ denoting the opposite sign of $\sigma$. The on-dot electron interactions are contained in the Liouvillian of impurities, $\mathcal{L} \star \equiv\left[H_{\mathrm{dot}}, \star\right]$.

In principle, the hierarchy of Eq. (6) extends to infinite levels $(L \rightarrow \infty)$ for quantum impurities involving electronelectron interactions, and hence, the solution of Eq. (6) must go through a systematic test to confirm its convergence versus $L$. In practice, usually, the HEOM results converge uniformly and rapidly with the increasing $L$, and hence, a relatively low $L$ (say, $L=4$ or 5 ) is often sufficient to yield quantitatively converged results.

It is a well-known limitation of the present HEOM approach that a higher $L$ is usually required to achieve numerical convergence at a lower temperature [51]. Consequently, the computational cost increases drastically as the temperature decreases. For a symmetric single-level Anderson model, the lowest temperature that can be accurately accessed by the present HEOM approach is around $0.1 T_{\mathrm{K}}\left(T_{\mathrm{K}}\right.$ is the Kondo temperature), using the computational resources at our disposal [51]. Therefore, to preserve a high numerical accuracy, in this work we focus on only the temperature range in which the present HEOM approach is capable of yielding fully converged results.

\section{RESULTS AND DISCUSSIONS}

\section{A. Thermopower calculated via the HEOM approach}

Thermopower is usually characterized by the Seebeck coefficient defined by $[60,61]$

$$
S_{\mathrm{def}} \equiv-\left(\frac{V_{T}}{\Delta T}\right)_{I=0}=\left(\frac{V}{\Delta T}\right)_{I=0} .
$$

It is measured by searching for the bias voltage $V$ that cancels $V_{T}$ [61]. With the HEOM approach, $S_{\text {def }}$ can be computed precisely by following the definition of Eq. (7): given a small temperature gradient $\Delta T$ applied across the two leads, we search for the voltage $V$, under which the voltage-driven current cancels exactly the thermally induced current (hence the net current is zero). The thermopower $S_{\text {def }}$ is thus obtained through the last equality of Eq. (7).

Obviously, the search for the appropriate amplitude of voltage $V$ to fulfill the vanishing current condition could be quite tedious. In contrast, it is computationally more convenient to focus on the linear regime, where the total electronic current is [62]

$$
I=G V+L_{T} \Delta T,
$$

and hence, the thermopower can be evaluated as

$$
S_{\text {linear }}=-L_{T} / G \text {. }
$$




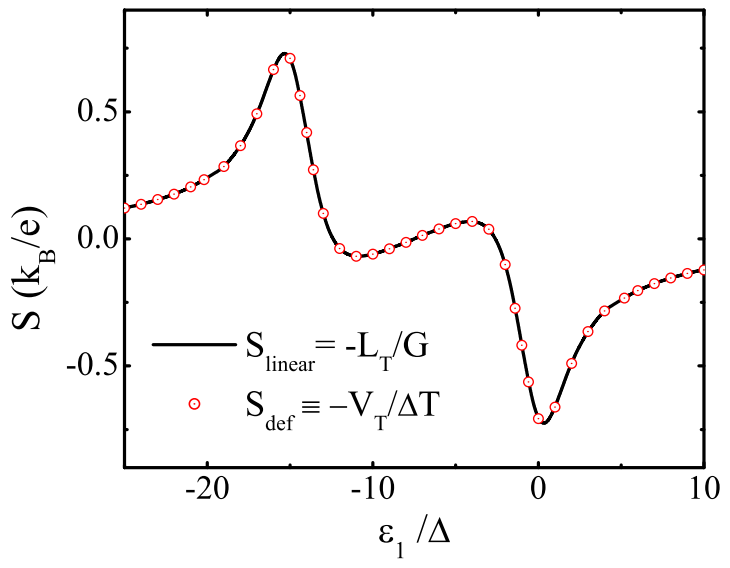

FIG. 1. (Color online) Thermopower of a single-level QD calculated with two schemes: (i) $S_{\text {def }}=\left.\left(\frac{V}{\Delta T}\right)\right|_{I=0}$ is computed by searching for the voltage $V$ that cancels the thermal current induced by a fixed $\Delta T=10^{-2} \Delta$. (ii) $S_{\text {linear }}=-L_{T} / G$ is obtained by computing the transport coefficients $L_{T}$ and $G$. The HEOM truncation level is $L=4$. The parameters of the QD are (in units of $\Delta$ ) $U=15, W=30$, and $T=0.2$.

The conductance $G=\left(\frac{\partial I}{\partial V}\right)_{\Delta T=0}$ and the coefficient $L_{T}=$ $\left(\frac{\partial I}{\partial \Delta T}\right)_{V=0}$ are evaluated by computing the electronic current response to a small temperature gradient $\Delta T$ and to a small bias voltage $V$, respectively.

Figure 1 compares the calculated $S_{\text {def }}$ and $S_{\text {linear }}$ of a single-level QD over a wide range of level energy $\epsilon_{1}$. Excellent agreement is clearly observed, which affirms that the above two schemes for computing the thermopower are numerically equivalent in the linear regime.

It is also known that $G$ and $L_{T}$ can be expressed by Landauer-type formulas [63]. This leads to the following expression of thermopower at equilibrium states (assuming $W$ is very large and setting $\hbar=k_{\mathrm{B}}=1$ ):

$$
S_{\text {Landauer }}=-\frac{1}{e T} \frac{\int d \omega(\omega-\mu) f^{\prime}(\omega) A(\omega)}{\int d \omega f^{\prime}(\omega) A(\omega)} .
$$

Here, $A(\omega)$ is the dot spectral function at equilibrium (see Sec. III B for more details), $f(\omega)$ is the Fermi function, and $f^{\prime}(\omega) \equiv \frac{\partial f(\omega)}{\partial \omega}$. Equation (10) is often approximated further to obtain the semiclassical Mott relation of

$$
S_{\text {Mott }}=-\frac{\pi^{2}}{3} \frac{k_{\mathrm{B}}^{2} T}{e} \frac{\partial \ln G}{\partial \epsilon}
$$

by assuming $A(\omega)$ is a slowly varying function of $\omega$ [64].

Figure 2(a) depicts the calculated conductance $G$ versus the level energy $\epsilon_{1}$ of a single-level QD. The curves exhibit three valleys, and each of them corresponds to an integer electron occupation number $N$ on the dot. In the valley of $N=1$, the increasing $G$ as $T$ decreases indicates the presence of S-Kondo resonance [55,65]. The Kondo temperature of the $\mathrm{QD}$ is $T_{\mathrm{K}}=8.4 \times 10^{-3} \Delta$ when the energy level aligns with the center of the $N=1$ valley $\left(\epsilon_{1}=\epsilon^{\text {eh }}=-U / 2\right.$, where $\epsilon^{\mathrm{eh}}$ is the electron-hole symmetry point) [66]. Limited by computational resources, it is difficult to access the $T \ll$ $T_{\mathrm{K}}$ regime with the present HEOM approach. Nevertheless,
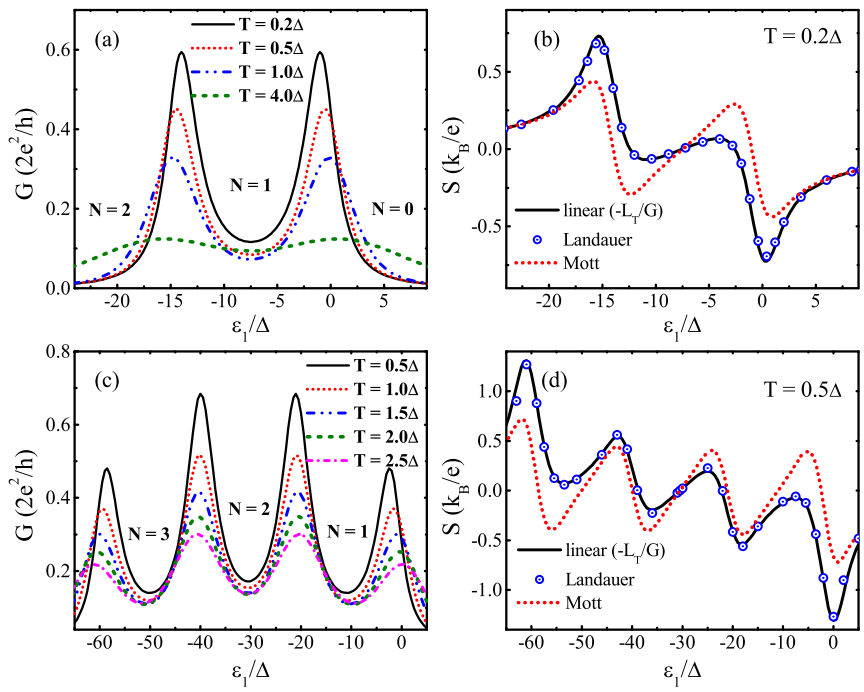

FIG. 2. (Color online) (a) $G$ and (b) $S$ vs $\epsilon_{1}$ for a single-level QD of $U=15 \Delta$ and $W=30 \Delta$. (c) $G$ and (b) $S$ vs $\epsilon_{1}$ for a two-level QD with the parameters of (in units of $\Delta$ ) $\delta \epsilon=\epsilon_{2}-\epsilon_{1}=1, U=20$, and $W=80$. The equilibrium chemical potentials of leads $\mu$ are set as zero energy.

although the Kondo resonance is strongly suppressed by the thermal fluctuation at $T>T_{\mathrm{K}}$, the surviving Kondo correlation still has significant influence on the QD electronic properties [53]. This is clearly affirmed by the increasing $G$ with decreasing $T$, as displayed in Fig. 2(a). Figure 2(b) compares the calculated $S_{\text {linear }}, S_{\text {Landauer }}$, and $S_{\text {Mott }}$ of the single-level QD. All the curves are antisymmetric with respect to the

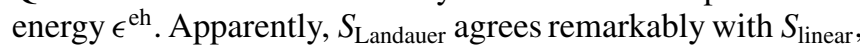
while $S_{\text {Mott }}$ exhibits severe deviation in the S-Kondo $(N=1)$ regime $[4,9]$.

Figure 2(c) displays the calculated $G$ versus $\epsilon_{1}$ of a two-level QD. The electron-hole symmetry point is now at the center of the $N=2$ valley, i.e., $\epsilon^{\text {eh }}=-(3 U+\delta \epsilon) / 2$. While the $N=1,2$, and 3 valleys all manifest apparent Kondo signatures ( $G$ increases with decreasing $T$ ), they are attributed to different types of Kondo correlations. The $N=2$ valley is dominated by the O-Kondo states [36], while the other two are associated with both S-Kondo and O-Kondo states [34]. The corresponding $S$ versus $\epsilon_{1}$ are shown in Fig. 2(d). As in the case of the single-level QD, $S_{\text {Landauer }}$ precisely reproduces $S_{\text {linear, }}$, while $S_{\text {Mott }}$ is some way off.

We emphasize that $S_{\text {linear }}$ and $S_{\text {Landauer }}$ are obtained via very different numerical procedures: while the computation of $S_{\text {linear }}$ is based on the evaluation of transport properties $G$ and $L_{T}$, $S_{\text {Landauer }}$ is completely determined by the dot spectral function $A(\omega)$. As shown in Fig. 2(d) [and also Fig. 3(c)], $S_{\text {linear }}$ and $S_{\text {Landauer }}$ agree remarkably with each other at a sufficiently high truncation level $L=4$. Meanwhile, we also show that such an excellent agreement is not reached at a lower $L$. As demonstrated in Figs. 3(a) and 3(b), the HEOM-calculated $S_{\text {linear }}$ and $S_{\text {Landauer }}$ deviate more from each other as $L$ decreases. This clearly indicates that only when the HEOM results converge with respect to $L$ can they be considered to be quantitatively accurate. 


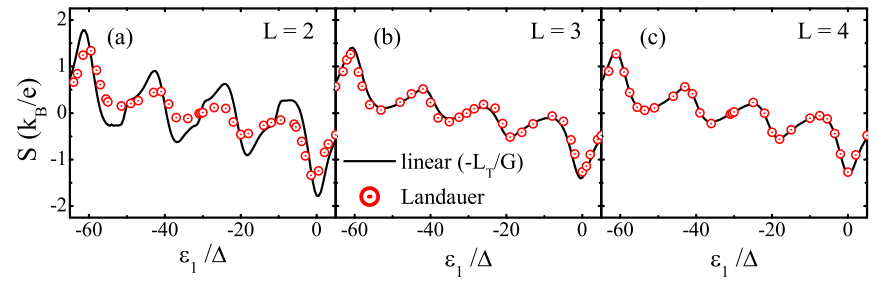

FIG. 3. (Color online) $S_{\text {linear }}$ and $S_{\text {Landauer }}$ of a two-level QD calculated by the HEOM approach at the truncation level of (a) $L=2$, (b) $L=3$, and (c) $L=4$. The parameters adopted are (in units of $\Delta$ ) $\delta \epsilon=1, U=20, W=80$, and $T=0.5$.

\section{B. Dot spectral function and Kondo spectral features}

The equilibrium spectral function $A_{i}(\omega)$ of the $i$ th dot level is associated with the retarded Green's function as follows:

$$
\begin{aligned}
A_{i}(\omega) & \equiv \frac{1}{2 \pi} \int d t e^{i \omega t}\left\langle\left\{\hat{a}_{i}(t), \hat{a}_{i}^{\dagger}(0)\right\}\right\rangle \\
& =-\frac{1}{\pi} \operatorname{Im}\left[G_{i i}^{r}(\omega)\right] .
\end{aligned}
$$

The computation of $A_{i}(\omega)$ and $G^{r}(\omega)$ in the framework of the HEOM method has been discussed extensively in Refs. [51,54].

Figure 4 depicts the HEOM-calculated $A(\omega)=A_{1}(\omega)$ of a single-level QD. Results obtained at different truncation levels $L$ are displayed. The $S$-Kondo state leads to a sharp spectral peak at $\omega=\mu$, and the dot level gives rise to the single-electron resonance peaks (Hubbard peaks) roughly at $\omega=\epsilon_{d}^{*}$ and $\omega=\epsilon_{d}^{*}+U$, with $\epsilon_{d}^{*}$ being the averaged renormalized level energy. Apparently, the two Hubbard peaks converge rapidly as $L$ increases, while the Kondo peak at $\omega=\mu=0$ converges more slowly. Note that the difference between the $L=4$ and $L=5$ curves is so small that the two curves cannot be distinguished by the naked eye. This affirms that the truncation at $L=4$ is sufficient to yield quantitatively accurate spectral function. Moreover, $A(\omega)$ is correctly normalized at any $L$, as

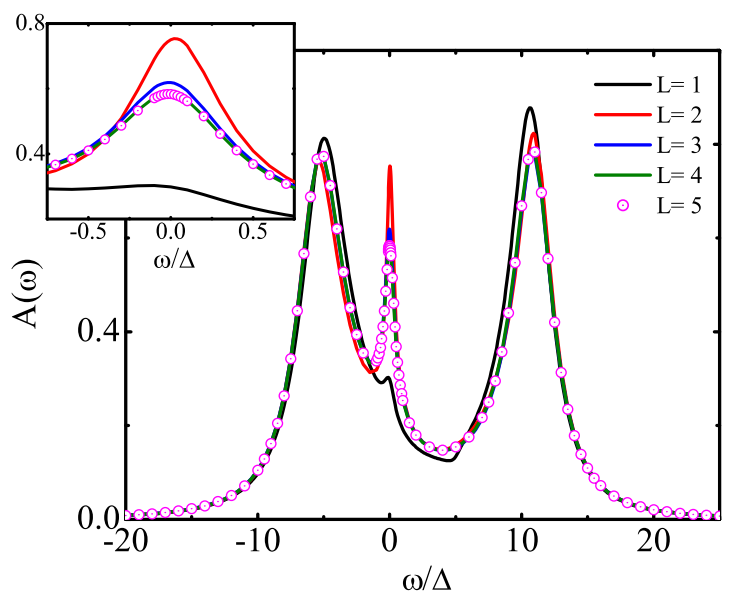

FIG. 4. (Color online) Spectral function of a single-level QD calculated with the HEOM approach at different truncation levels. The inset magnifies the Kondo spectral peak around $\omega=0$. The parameters of the QD are (in units of $\Delta$ ) $\epsilon_{1}=-5, U=15, W=30$, and $T=0.2$.
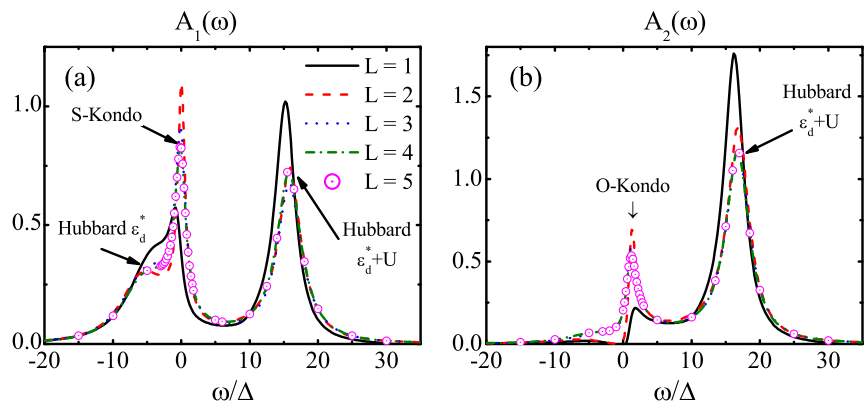

FIG. 5. (Color online) (a) $A_{1}(\omega)$ and (b) $A_{2}(\omega)$ of a two-level QD calculated at different truncation levels $L$. The parameters adopted are (in units of $\Delta$ ) $\epsilon_{1}=-5.5, \delta \epsilon=1, U=20, W=80$, and $T=0.5$.

$\int_{-\infty}^{+\infty} A(\omega) d \omega=1$ is satisfied to numerical precision. Figure 4 clearly affirms that the HEOM approach can accurately capture the Kondo spectral peaks at a relatively low $L$.

We then examine a two-level QD with a small level spacing $\delta \epsilon=\epsilon_{2}-\epsilon_{1}=\Delta$. The QD is in the $N=1$ regime with $\epsilon_{1}=$ $-5.5 \Delta$. Figures $5(\mathrm{a})$ and 5 (b) display the calculated $A_{i}(\omega)$ for $i=1$ and 2 , respectively. The total dot spectral function is $A(\omega)=\frac{1}{2}\left[A_{1}(\omega)+A_{2}(\omega)\right]$. Here, the factor $\frac{1}{2}$ preserves the normalization condition of $\int_{-\infty}^{+\infty} A(\omega) d \omega=1$. From Fig. 5, it is evident that $A(\omega)$ of a two-level QD also converges quantitatively at $L=4$ at the temperature $T=0.5 \Delta$.

As shown in Fig. 5(a), an S-Kondo resonance peak is present at $\omega=\mu=0$ at $T=0.5 \Delta$. The S-Kondo peak is contributed completely by level 1 . This is because $\epsilon_{2}>\epsilon_{1}$, so that level 1 is close to half filling, while level 2 is almost vacant. Aside from the S-Kondo peak, the interlevel Coulomb interaction is expected to give a pair of satellite peaks at $\omega=\mu \pm \delta \epsilon[33,67]$. In Fig. 5(b) we observe an O-Kondo peak at $\omega=\mu+\delta \epsilon$ contributed by level 2; however, the other O-Kondo peak expected to appear at $\omega=\mu-\delta \epsilon$ is absent.

The presence (and absence) of S-Kondo and O-Kondo peaks can be understood as follows. For the QD studied in Fig. 5, an electron may hop from a certain lead onto the empty level 2, accompanied by another electron hopping from level 1 to the counter lead $[33,34]$. The net effect is that an electron transfers across the dot and a second electron gets excited locally from level 1 to level 2 . Through such a virtual two-electron process, level 2 gains an energy of $\epsilon_{2}-\epsilon_{1}=\delta \epsilon$. Therefore, the virtual process leads to a resonance peak (the O-Kondo peak) at $\omega=\mu+\delta \epsilon$ in $A_{2}(\omega)$. Meanwhile, level 1 is near half filling, and thus, an electron may hop from level 1 to a certain lead, accompanied by another electron entering into the dot from the counter lead. Because $\epsilon_{1}<\epsilon_{2}$, it is energetically more favorable for the incoming electron to occupy level 1 . Note that the spin of the incoming electron is opposite that of the leaving electron due to Pauli exclusion [34]. Such a two-electron cotunneling process results in an effective spin flip on level 1 and thus gives rise to the $\mathrm{S}$-Kondo peak at $\omega=\mu$ in $A_{1}(\omega)$. This also explains why the expected O-Kondo peak at $\omega=\mu-\delta \epsilon$ does not show up: the corresponding virtual process is energetically unfavorable in a competition with the $\mathrm{S}$-Kondo resonance.

Figure 6 displays the distribution of Kondo spectral peaks for the same two-level QD studied in Fig. 5. The three 


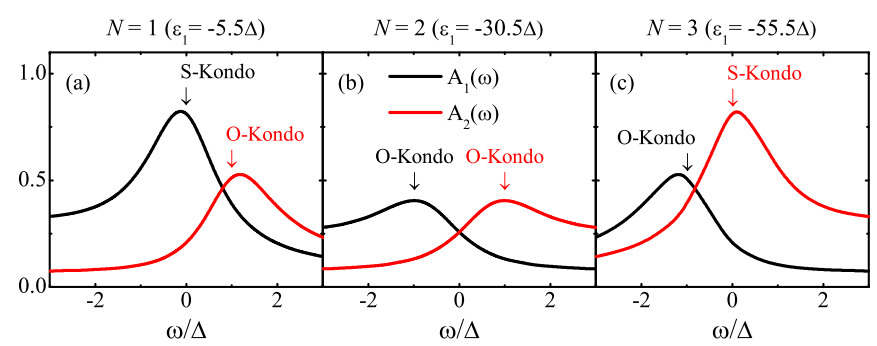

FIG. 6. (Color online) Calculated $A_{i}(\omega)(i=1,2)$ of a two-level QD in the (a) $N=1$, (b) $N=2$, and (c) $N=3$ regimes. The parameters adopted are (in units of $\Delta$ ) $\delta \epsilon=1, U=20$, and $T=0.5$.

panels explore the $N=1,2$, and 3 regimes (corresponding to different values of $\epsilon_{1}$ ), respectively. The $N=1$ regime has been discussed in detail in the previous paragraph.

In the $N=2$ regime, level 1 is almost fully occupied, while the occupation number in level 2 remains rather small. Therefore, the virtual two-electron process associated with level 2 is essentially identical to that in the case of $N=1$. Indeed, $A_{2}(\omega)$ depicted in Fig. 6(b) exhibits an O-Kondo peak at $\omega=\mu+\delta \epsilon$, the same as in Fig. 6(a). However, the virtual process associated with level 1 is distinctly different from the case of $N=1$. With an electron hopping from level 1 to a certain lead, the incoming electron from the counter lead cannot populate onto level 1 because level 1 has already been occupied by an opposite-spin electron. Therefore, the incoming electron has to occupy the vacant level 2. Through such a virtual process, level 1 effectively loses an energy of $\delta \epsilon$, and consequently, $A_{1}(\omega)$ exhibits an O-Kondo peak at $\omega=\mu-\delta \epsilon$.

In the $N=3$ regime, level 1 is almost fully occupied, while level 2 is close to half filling. The virtual two-electron process associated with levels 1 and 2 can be analyzed like those as above. These electron cotunneling events give rise to the O-Kondo peak at $\omega=\mu-\delta \epsilon$ in $A_{1}(\omega)$ and the S-Kondo peak at $\omega=\mu$ in $A_{2}(\omega)$, as shown in Fig. 6(c).

The virtual two-electron process can also be interpreted as cotunneling of holes. For instance, the $N=3$ regime corresponds to the situation of single-hole occupation on level2 . Because of the electron-hole symmetry, the distribution of Kondo spectral peaks in the $N=3$ regime forms a mirror image of that in the $N=1$ regime [see Figs. 6(a) and 6(c)].

\section{Relation between dot spectral function and thermopower}

The remarkable agreement between $S_{\text {Landauer }}$ and $S_{\text {linear }}$ in Fig. 2 verifies that Eq. (10) is highly accurate. It is inferred from Eq. (10) that $S$ is completely determined by the equilibrium-state dot spectral function $A(\omega)$. Since different types of electronic states have distinctive spectral features, their influence on the thermopower can be unraveled through Eq. (10).

The sign of $S$ is of particular importance because it predicts the direction of thermal electric current driven by a given temperature gradient across the two leads. By definition, $A(\omega) \geqslant 0$ at all $\omega$, and $f^{\prime}(\omega)$ is negative around $\mu$ and almost zero elsewhere. Therefore, $f^{\prime}(\omega)$ defines a thermal activation window. The width of the window $\Omega$ is determined solely by the temperature $T$ via the analytic form of $f^{\prime}(\omega)$. The spectral

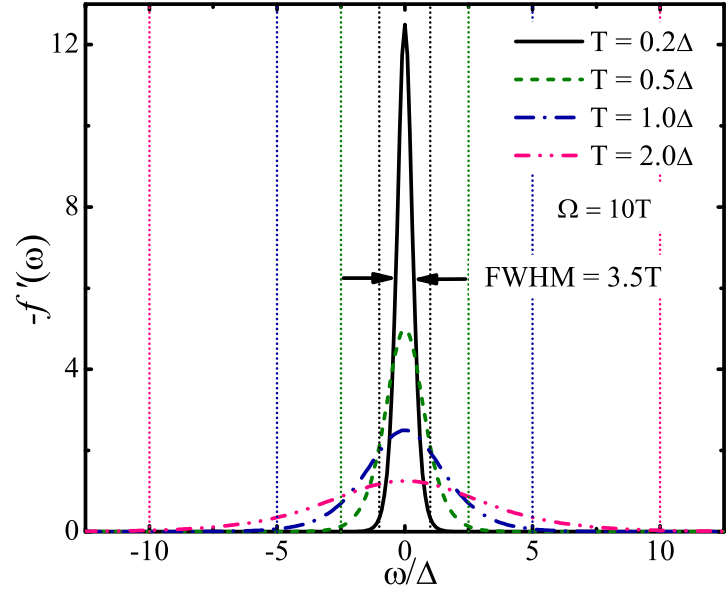

FIG. 7. (Color online) The line shapes of $-f^{\prime}(\omega)$ at various $T$. The boundaries of the thermal activation window are indicated by dotted vertical lines.

density $A(\omega)$ outside this window has almost no contribution to $S$.

As depicted in Fig. $7,-f^{\prime}(\omega) \geqslant 0$ over the entire range of $\omega$, and it exhibits a single peak at $\omega=\mu=0$. The full width at half maximum (FWHM) of the peak is $2 \ln (3+2 \sqrt{2}) T \simeq$ $3.5 T$, and thus, a higher $T$ should be associated with a larger thermal activation window. We choose $\Omega=10 T$. Such an $\Omega$ is sufficiently large for accurate evaluation of $S$ through Eq. (10). The boundaries of the thermal activation window defined by the width $\Omega$ are displayed in Fig. 7.

The Landauer-type formula of Eq. (10) can be recast as follows:

$$
\begin{aligned}
S & \simeq-\frac{1}{e T} \frac{\int_{\mu-\Omega / 2}^{\mu+\Omega / 2} d \omega(\omega-\mu) f^{\prime}(\omega) A(\omega)}{\int d \omega f^{\prime}(\omega) A(\omega)} \\
& =-\frac{\Omega}{e T} \frac{\left(\omega_{I}-\mu\right) f^{\prime}\left(\omega_{I}\right) A\left(\omega_{I}\right)}{\int d \omega f^{\prime}(\omega) A(\omega)} .
\end{aligned}
$$

Here, the first equality holds because $f^{\prime}(\omega)$ is essentially zero outside the thermal activation window $\left(\mu-\frac{\Omega}{2}, \mu+\frac{\Omega}{2}\right)$. The second equality uses the mean value theorem, with $\omega_{I}$ being a certain energy within the thermal activation window. The relative weight of $A(\omega)$ around $\mu$ is thus quantified by the value of $\omega_{I}$; if $A(\omega)$ is, overall, larger in the range of $\omega \in\left(\mu, \mu+\frac{\Omega}{2}\right)$ than in $\left(\mu-\frac{\Omega}{2}, \mu\right)$, we have $\omega_{I}>\mu$ and $S<0$ and vice versa.

The physical origin of S-Kondo and O-Kondo spectral peaks has been discussed in Sec. III B. In the following, we analyze the spectral features of various types of electronic states in a two-level QD and unravel their contributions to the thermopower using Eq. (13).

We choose $T_{\mathrm{L}}$ to be a low temperature at which both the $\mathrm{S}$-Kondo and O-Kondo correlations are present and $T_{\mathrm{H}}$ to be a high temperature at which all the Kondo correlations are completely destroyed.

In the absence of external fields, the S-Kondo state leads to a sharp spectral peak at $\omega=\mu$; see Fig. 8(a) for a schematic diagram of $A(\omega)$ at $T_{\mathrm{L}}$. The O-Kondo states emerge only when the dot levels are degenerate or nearly degenerate. They give rise to a pair of satellite peaks at $\omega=\mu \pm \delta \epsilon[33,67]$. Since the 

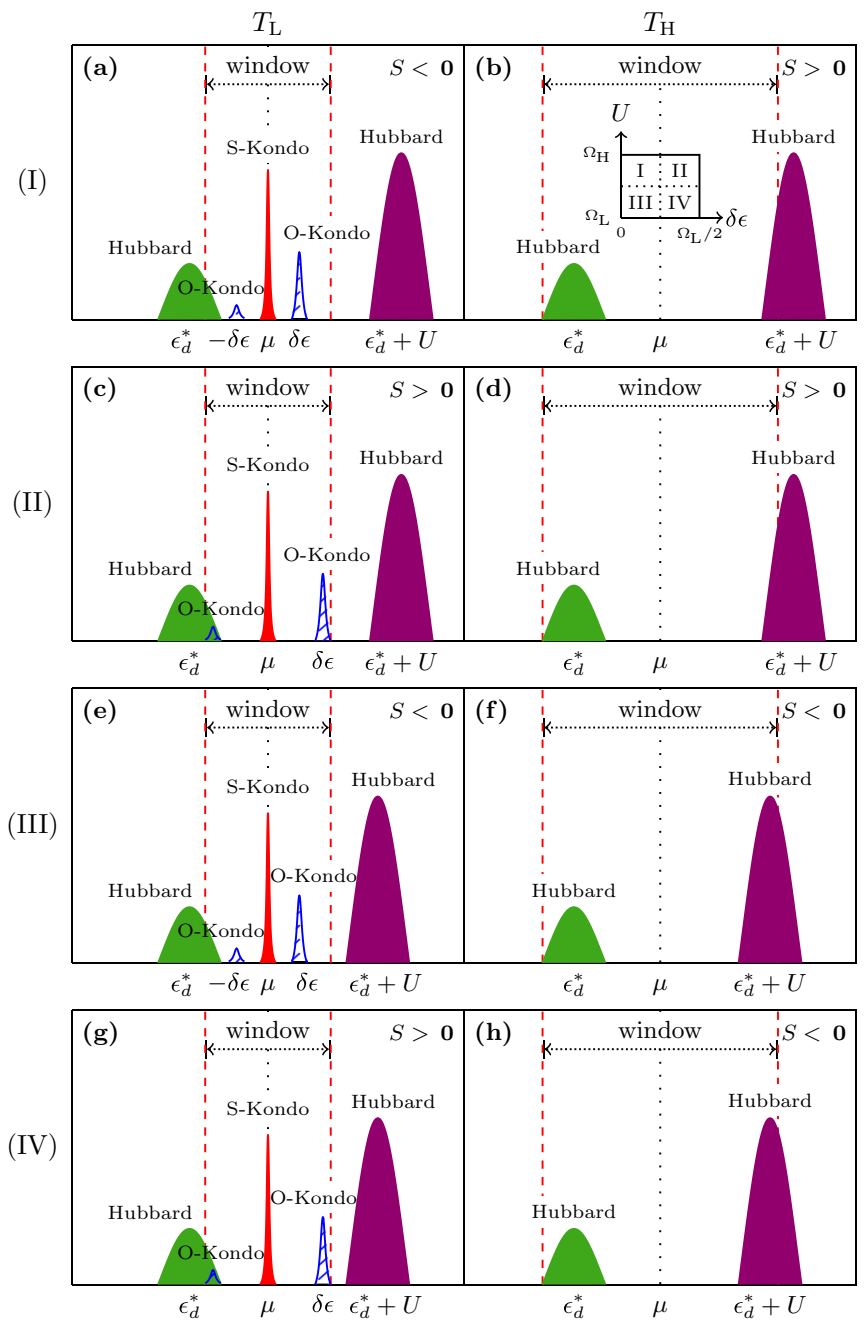

FIG. 8. (Color online) Schematic diagram of the dot spectral function of a two-level QD in the $N=1$ region. The spectral peaks originating from the various electronic states are depicted. The left and right columns correspond to temperatures $T_{\mathrm{L}}$ and $T_{\mathrm{H}}$, respectively. Each row represents a scenario corresponding to a certain range of parameters $(\delta \epsilon, U)$, as illustrated in the inset in (b). The thermal activation window and the resultant sign of $S$ are also shown in each panel.

Kondo peaks locate at or near $\mu$, they have crucial influence on the value of $S$ at $T_{\mathrm{L}}$. Note that $T_{\mathrm{L}}$ need not be lower than $T_{\mathrm{K}}$; although the Kondo peak heights are greatly suppressed by thermal fluctuation, the peak positions and line shapes can be largely preserved even at $T_{\mathrm{L}}>T_{\mathrm{K}}$ [53]. Aside from the Kondo peaks, the dot levels also give rise to the single-electron resonance peaks (Hubbard peaks) roughly at $\omega=\epsilon_{d}^{*}$ and $\omega=\epsilon_{d}^{*}+U$, with $\epsilon_{d}^{*}$ being the averaged renormalized level energy. In contrast, all the Kondo peaks vanish completely at $T_{\mathrm{H}}$, while the Hubbard peaks are little affected by the variation of temperature [see Fig. 8(b)]. Therefore, it is the Hubbard peaks that dominate the $S$ at $T_{\mathrm{H}}$.

Using the above analysis, we now elucidate how $A(\omega)$ determines the sign of $S$ for a two-level QD. We focus on the $N=1$ regime $\left(\mu-\frac{U}{2}<\epsilon_{1}<\mu\right)$, in which both $S$-Kondo and O-Kondo correlations take effect at $T_{\mathrm{L}}$. In particular, we consider the range of $0<\delta \epsilon<\frac{\Omega_{\mathrm{L}}}{2}$ and $\Omega_{\mathrm{L}}<U<\Omega_{\mathrm{H}}$, where $\Omega_{\mathrm{L}}\left(\Omega_{\mathrm{H}}\right)$ is the width of the thermal activation window at temperature $T_{\mathrm{L}}\left(T_{\mathrm{H}}\right)$. Such a $(\delta \epsilon, U)$ subspace is further divided into four zones [see the inset of Fig. 8(b)]. Each zone corresponds to a distinctive scenario and is illustrated by a specific row in Fig. 8.

Zone (I) is associated with a small $\delta \epsilon$ and a large $U$. As shown in Fig. 8(a), at $T_{\mathrm{L}}$ the S-Kondo and O-Kondo peaks locate within the thermal activation window, while the Hubbard peaks stay outside (their tails may extend into the window). The S-Kondo peak is nearly symmetric around $\mu$, and it thus has rather minor effects on the sign of $S$. Because the QD is in the $N=1$ regime, the O-Kondo peak at $\omega=\mu+\delta \epsilon$ is much higher than that at $\omega=\mu-\delta \epsilon$, as demonstrated in Fig. 5. This is because the virtual two-electron process associated with the latter is energetically unfavorable compared with the S-Kondo resonance; see the related remarks in Sec. III B. Therefore, $A(\omega)$ is, overall, larger in the $\omega>\mu$ half of the window. It is thus inferred from Eq. (13) that $\omega_{I}>\mu$ and $S<0$. The situation of $T_{\mathrm{H}}$ is illustrated by Fig. 8(b). At $T_{\mathrm{H}}$ all the Kondo peaks vanish completely. Since $\epsilon_{d}^{*} \sim \epsilon_{1}>\mu-\frac{U}{2}$, the energy $\epsilon_{d}^{*}$ is closer to $\mu$ than $\epsilon_{d}^{*}+U$. Consequently, the Hubbard peak at $\omega=\epsilon_{d}^{*}$ lies partly within the window, while the Hubbard peak at $\omega=\epsilon_{d}^{*}+U$ remains largely outside the window. Therefore, $A(\omega)$ is, overall, larger in the $\omega<\mu$ half of the window, which implies that $\omega_{I}<\mu$ and $S>0$. Altogether, our analysis predicts that the QDs in zone (I) will undergo a sign change of $S$ from negative to positive as the temperature increases from $T_{\mathrm{L}}$ to $T_{\mathrm{H}}$.

Zone (IV) corresponds to a large $\delta \epsilon$ and a small $U$. As depicted in Fig. $8(\mathrm{~g})$, at $T_{\mathrm{L}}$ the O-Kondo peaks locate close to the edge of the window. Therefore, the sign of $S$ is trivially related to the Kondo peaks and is largely determined by the distribution of Hubbard peaks. With a small $U$, some portion of the Hubbard peak at $\omega=\epsilon_{d}^{*}$ extends into the window, while the peak at $\omega=\epsilon_{d}^{*}+U$ stays mainly outside of it; we thus have $\omega_{I}<\mu$ and $S>0$. The situation of $T_{\mathrm{H}}$ is illustrated in Fig. 8(h), where both Hubbard peaks have substantial distribution within the window. Because the peak at $\epsilon_{d}^{*}+U$ is much higher than that at $\epsilon_{d}^{*}, A(\omega)$ is, overall, larger in the $\omega>\mu$ half of the window, which implies that $\omega_{I}>\mu$ and $S<0$. Altogether, it is predicted that the QDs in zone (IV) will experience a sign change of $S$ from positive to negative as the temperature increases from $T_{\mathrm{L}}$ to $T_{\mathrm{H}}$.

For the other two zones covering the rest of the $(\delta \epsilon, U)$ subspace of interest, the relation between $A(\omega)$ and $S$ can be analyzed similarly. For QDs in zone (II) [zone (III)], $S$ is predicted to retain a positive (negative) sign throughout the temperature range $T_{\mathrm{L}}<T<T_{\mathrm{H}}$.

We point out that for a QD in any of the four zones, the Hubbard peak at $\omega=\epsilon_{d}^{*}+U$ is always much higher than that at $\omega=\epsilon_{d}^{*}$. In particular, the ratio of the area under the former peak to that under the latter is roughly $3: 1$. This is because we focused on the $N=1$ regime, and hence, the average occupation number on a dot level (per spin) is around $\frac{1}{4}$. Consequently, we should have $\int_{-\infty}^{\mu} A(\omega) d \omega \approx \frac{1}{4}$ and $\int_{\mu}^{\infty} A(\omega) d \omega \approx \frac{3}{4}$. This is verified by the HEOM-calculated $A(\omega)$ shown in Figs. 9-12.

Figure 8 thus provides a comprehensive picture which clearly unravels the effects of the various electronic states on 


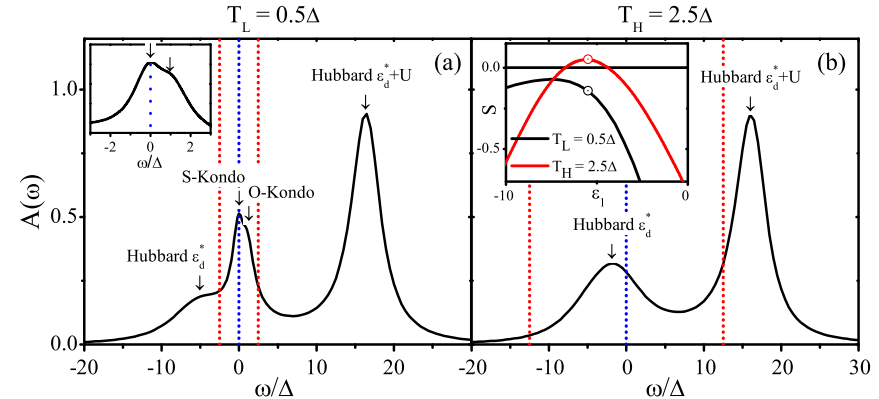

FIG. 9. (Color online) Calculated $A(\omega)$ of a two-level QD in the $N=1$ regime $\left(\epsilon_{1}=-5.5 \Delta\right)$ with $(\delta \epsilon, U)=(\Delta, 20 \Delta)$ at (a) $T_{\mathrm{L}}=$ $0.5 \Delta$ and (b) $T_{\mathrm{H}}=2.5 \Delta$. The various spectral peaks are marked by arrows, and the thermal activation window is indicated by dashed lines. The inset in (a) magnifies $A(\omega)$ around $\mu=0$ at $T_{\mathrm{L}}$, while the inset in (b) depicts the calculated $S$ vs $\epsilon_{1}$.

the thermopower. In Sec. III D we verify such a picture using the calculated $A(\omega)$ and $S$ obtained with the HEOM approach.

\section{Validation of Fig. 8 using the HEOM approach}

Figures 9(a) and 9(b) depict the calculated $A(\omega)$ of a two-level QD at $T_{\mathrm{L}}=0.5 \Delta$ and $T_{\mathrm{H}}=2.5 \Delta$, respectively. The dot parameters are $\delta \epsilon=\Delta$ and $U=20 \Delta$. Since $\delta \epsilon$ is much smaller than $\frac{\Omega_{\mathrm{L}}}{2}$ while $U$ is close to $\Omega_{\mathrm{H}}$, the QD is presumably in zone (I). As can be seen from Fig. 9, the distribution of the various spectral peaks in the calculated $A(\omega)$ agrees remarkably with the schematic diagram depicted in Figs. 8(a) and 8 (b). In particular, the O-Kondo peak at $\omega=\mu+\delta \epsilon$ and the Hubbard peak at $\omega=\epsilon_{d}^{*}$ contribute dominantly to the sign of $S$ at $T_{\mathrm{L}}$ and $T_{\mathrm{H}}$, respectively. This thus affirms our designation that the QD is in zone (I). Our above analysis has predicted a sign change of $S$ from negative to positive as $T$ increases from $T_{\mathrm{L}}$ to $T_{\mathrm{H}}$. Such a sign change is, indeed, observed in the HEOM-calculated data [see the inset of Fig. 9(b)].

Figures 10(a) and 10(b) depict $A(\omega)$ of another QD with $(\delta \epsilon, U)=(2 \Delta, 20 \Delta)$ at $T_{\mathrm{L}}=0.5 \Delta$ and $T_{\mathrm{H}}=2.5 \Delta$, respectively. Such a QD belongs to zone (II). Clearly, the O-Kondo peak locates near the edge of the thermal activation window because of the large $\delta \epsilon=2 \Delta$ [see Fig. 10(a)]. The sign of $S$ is thus determined mainly by the distribution of the

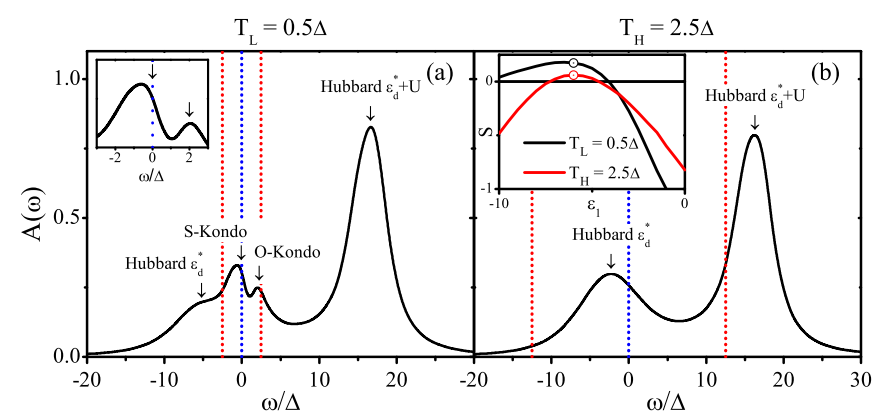

FIG. 10. (Color online) Calculated $A(\omega)$ of a two-level QD in the $N=1$ regime $\left(\epsilon_{1}=-6 \Delta\right)$ with $(\delta \epsilon, U)=(2 \Delta, 20 \Delta)$ at (a) $T_{\mathrm{L}}=$ $0.5 \Delta$ and (b) $T_{\mathrm{H}}=2.5 \Delta$. The inset in (a) magnifies $A(\omega)$ around $\mu=0$ at $T_{\mathrm{L}}$, while the inset in (b) depicts the calculated $S$ vs $\epsilon_{1}$.

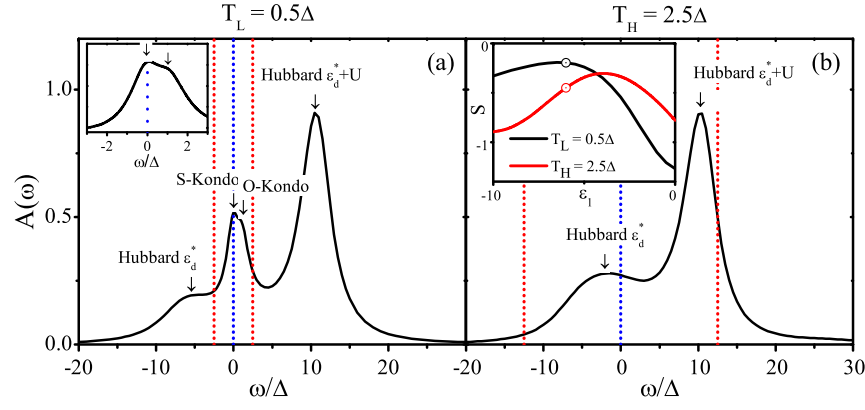

FIG. 11. (Color online) Calculated $A(\omega)$ of a two-level QD in the $N=1$ regime $\left(\epsilon_{1}=-6 \Delta\right)$ with $(\delta \epsilon, U)=(\Delta, 15 \Delta)$ at (a) $T_{\mathrm{L}}=0.5 \Delta$ and (b) $T_{\mathrm{H}}=2.5 \Delta$. The inset in (a) magnifies $A(\omega)$ around $\mu=0$ at $T_{\mathrm{L}}$, while the inset in (b) depicts the calculated $S$ vs $\epsilon_{1}$.

Hubbard peaks. In the temperature range of $T_{\mathrm{L}}<T<T_{\mathrm{H}}$, the Hubbard peak at $\omega=\epsilon_{d}^{*}+U$ always resides outside the window (because of the large $U$ ), while the Hubbard peak at $\omega=\epsilon_{d}^{*}$ has a significant distribution within the window. Therefore, $A(\omega)$ is, overall, larger in the $\omega<\mu$ half of the window, leading to a positive $S$ [see the inset in Fig. 10(b)].

Figures 11(a) and 11(b) depict the calculated $A(\omega)$ of the QD with $(\delta \epsilon, U)=(\Delta, 15 \Delta)$ at $T_{\mathrm{L}}=0.5 \Delta$ and $T_{\mathrm{H}}=2.5 \Delta$, respectively. At temperature $T_{\mathrm{L}}$, the O-Kondo peak at $\omega=$ $\mu+\delta \epsilon$ lies within the thermal activation window, while the Hubbard peaks mainly stay outside of it, as depicted in Fig. 11(a). Therefore, $A(\omega)$ is, overall, larger in the $\omega>\mu$ half of the window, leading to a negative $S$ at $T_{\mathrm{L}}$. At $T_{\mathrm{H}}$ both Hubbard peaks are within the window. Note that the Hubbard peak at $\omega=\epsilon_{d}^{*}+U$ is significantly higher than that at $\omega=\epsilon_{d}^{*}$, as depicted in Fig. 11(b). Therefore, $A(\omega)$ is also larger in the $\omega>\mu$ half of the window, and the negative sign of $S$ is preserved. This thus affirms our designation that the QD is in zone (III).

We then examine a QD with a larger $\delta \epsilon=2 \Delta$ and a smaller $U=15 \Delta$. The calculated $A(\omega)$ at the same $T_{\mathrm{L}}$ and $T_{\mathrm{H}}$ are displayed in Figs. 12(a) and 12(b), respectively. The distributions of the various spectral peaks conform well with the schematic diagrams in Figs. 8(g) and 8(h), indicating that such a QD should belong to zone (IV). Indeed, the calculated $S$ exhibits a sign change from positive to negative as $T$ varies from $T_{\mathrm{L}}$ to $T_{\mathrm{H}}$. This thus verifies our prediction on the thermoelectric behavior of the QDs in zone (IV).

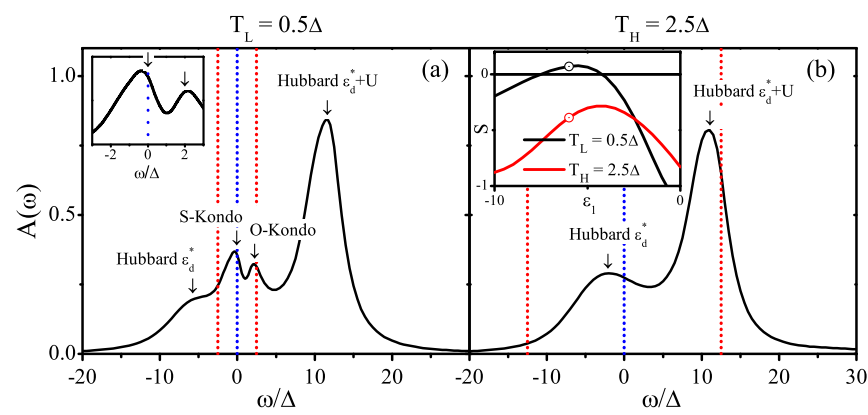

FIG. 12. (Color online) Calculated $A(\omega)$ of a two-level QD in the $N=1$ regime $\left(\epsilon_{1}=-6 \Delta\right)$ with $(\delta \epsilon, U)=(2 \Delta, 15 \Delta)$ at (a) $T_{\mathrm{L}}=$ $0.5 \Delta$ and (b) $T_{\mathrm{H}}=2.5 \Delta$. The inset in (a) magnifies $A(\omega)$ around $\mu=0$ at $T_{\mathrm{L}}$, while the inset in (b) depicts the calculated $S$ vs $\epsilon_{1}$. 


\section{CONCLUDING REMARKS}

To conclude, we have elucidated the underlying relations between the thermopower and the characteristic spectral features of two-level QDs. In particular, we have explored how the various types of electronic states (including the spin-Kondo states, orbital-Kondo states, and single-electron resonant states) influence the thermopower. With these insights, we have exemplified an effective and viable way to control the sign of thermopower of Kondo-correlated QDs. This is realized by tuning the temperature and by selecting the appropriate level spacing $\delta \epsilon$ and Coulomb repulsion strength $U$. Our understandings and findings provide useful insights into controlling the direction of electric (heat) current through a QD by applying a temperature (voltage) gradient across the two coupling leads. This may have important implications for novel thermoelectric applications of QDs.

This work has focused on the linear response regime in which the temperature gradient or bias voltage applied across the dot is rather small. It will be even more interesting and challenging to investigate the thermoelectric properties of Kondo-correlated QDs under a large bias voltage or temperature gradient. The HEOM method provides a unified approach for characterizing equilibrium and nonequilibrium properties of quantum impurity systems, and hence, it is potentially useful for studies of far-from-equilibrium QDs. Work along this direction is underway.

\section{ACKNOWLEDGMENTS}

The support from the Natural Science Foundation of China (Grants No. 21033008, No. 21233007, No. 21303175, and No. 21322305), the Strategic Priority Research Program (B) of the CAS (XDB01020000), and the Hong Kong University Grants Committee (Grant No. AoE/P-04/08-2) and Research Grants Council (Grant No. 605012) is gratefully appreciated. L.Y. and X.Z. thank Prof. M. Di Ventra for the stimulating discussions.

\section{APPENDIX: VALIDITY OF THE HEOM APPROACH FOR TREATING STRONGLY CORRELATED QUANTUM DOTS}

The numerical accuracy of the HEOM approach has been examined carefully and comprehensively in many of our previous works, such as in Refs. [51-54]. In particular, the correct Kondo scaling behavior and the analytic logarithmic Kondo tail have been recovered with the HEOM calculations [51].

A wide range of electronic properties of strongly correlated quantum impurity models has been calculated with the HEOM approach, and the resulting data agree remarkably with those obtained with the high-level numerical renormalization group (NRG) methods. These include the spectral function (Fig. 2 and supplemental Fig. S9 in Ref. [51]), the steady-state current under bias voltages (Fig. S4 in the Supplemental Material of Ref. [53]), and the local magnetic susceptibility (Fig. 4 in Ref. [52]).

In this appendix, we provide more evidence of the numerical validity and accuracy of the HEOM approach.

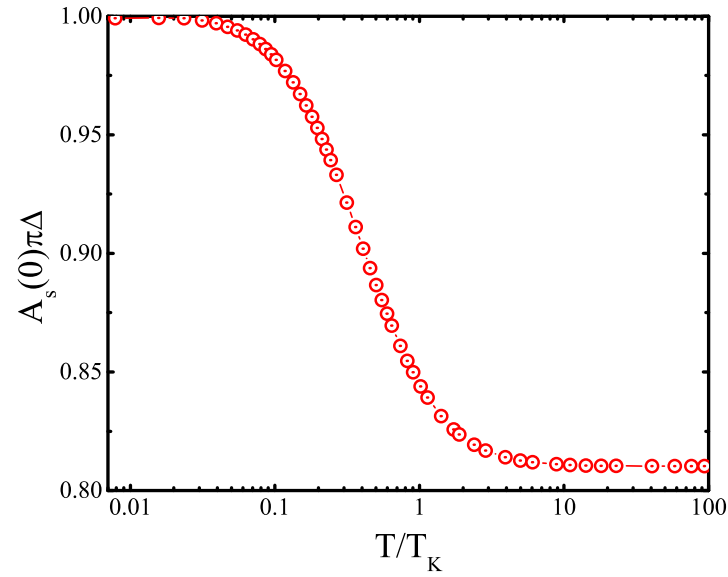

FIG. 13. (Color online) Calculated $A_{s}(\mu)$ as a function of $T / T_{\mathrm{K}}$. The QD parameters are (in units of $\Delta$ ) $U=-2 \epsilon_{1}=1.6$ and $W=20$.

\section{Friedel sum rule}

The Friedel sum rule implies that the following relation should hold exactly for a QD at zero temperature $[68,69]$ :

$$
A_{s}(\mu)=\frac{\sin ^{2}\left(\pi n_{s}\right)}{\pi \Delta},
$$

where $A_{s}(\mu)$ is the spectral density of spin-s electrons at the chemical potential $\mu$ and $n_{s}$ is the occupation number of spin- $s$ electrons on the dot.

We now examine the relation of Eq. (A1) on a symmetric Anderson QD. The HEOM-calculated $A_{s}(\mu) \equiv A_{\uparrow}(\mu)=$ $A_{\downarrow}(\mu)$ versus the scaled temperature $T / T_{\mathrm{K}}$ is shown in Fig. 13, with the Kondo temperature $T_{\mathrm{K}}$ evaluated as follows [66]:

$$
T_{\mathrm{K}}=\sqrt{\frac{U \Delta}{2}} e^{-\pi U / 8 \Delta+\pi \Delta / 2 U} .
$$

At the electron-hole symmetry point $\epsilon_{1}=-U / 2$, the QD is exactly half filled $\left(n_{s}=\frac{1}{2}\right)$. It is clearly demonstrated in Fig. 13 that, as $T$ decreases, $A_{s}(\mu)$ correctly approaches $\frac{1}{\pi \Delta}$. This thus verifies that the prediction of Eq. (A1) is accurately achieved with the HEOM approach.

\section{Conductance of single-level Kondo QDs at $\boldsymbol{T} \rightarrow \mathbf{0}$}

In Ref. [51], it is shown that the HEOM approach reproduces the correct Kondo scaling behavior through the calculated zero-bias conductance $d I / d V$ versus $T / T_{\mathrm{K}}$. Below, we verify that the HEOM approach yields the correct conductance in the limit of $T \rightarrow 0$.

At low temperatures, the relation between the zero-bias conductance $G$ and the temperature $T$ can be described by the empirical Goldhaber-Gordon form [70,71]:

$$
G(T)=G_{0}\left[1+\left(2^{\frac{1}{s}}-1\right)\left(\frac{T}{T_{\mathrm{K}}}\right)^{2}\right]^{-s},
$$

where $T_{\mathrm{K}}$ is the Kondo temperature, $G_{0}=2 e^{2} / h$ is the conductance quantum, and $s$ is a scaling constant. Equation (A3) can be rearranged as

$$
\left(\frac{G}{G_{0}}\right)^{-\frac{1}{s}}=1+\left(2^{\frac{1}{s}}-1\right)\left(\frac{T}{T_{\mathrm{K}}}\right)^{2} .
$$




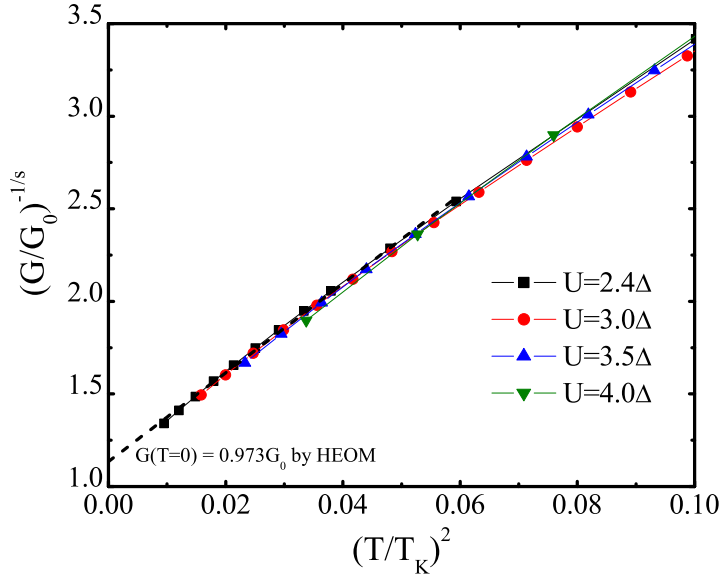

FIG. 14. (Color online) $\left(G / G_{0}\right)^{-1 / s}$ vs $\left(T / T_{\mathrm{K}}\right)^{2}$. The parameters adopted are $\epsilon_{1}=-U / 2$ and $W=20 \Delta$. The scaling constant is $s=$ 0.217 .

We then calculate $G$ versus $T$ for a series of single-level QDs with the HEOM approach. The resulting data are analyzed as follows: (i) Assign a certain value to $s$. (ii) Plot $\left(G / G_{0}\right)^{-1 / s}$ versus $\left(T / T_{\mathrm{K}}\right)^{2}$ and fit the data to a straight line. Here, $T_{\mathrm{K}}$ is evaluated via Eq. (A2). (iii) Extract the value of $s$ by relating the slope of the fitted line to $\left(2^{1 / s}-1\right)$. (iv) Repeat step (i) until the value of $s$ converges.

Figure 14 plots $\left(G / G_{0}\right)^{-1 / s}$ versus $\left(T / T_{\mathrm{K}}\right)^{2}$ with the converged $s=0.217$. Such a value of $s$ agrees well with previous theoretical and experimental studies [70-72]. In Fig. 14, the calculated data for the various QDs with different values of $U$ fall on the same straight line. This clearly affirms that the Kondo scaling behavior is, indeed, "universal." Moreover, although it is very difficult to calculate $G(T=0)$ with the present HEOM approach (the computational cost increases drastically as $T$ decreases) [48], the value of $G(T=$ 0 ) can be estimated by extrapolating the fitted straight line to $T=0$ and measuring the intercept. In doing so, we obtain $G(T=0) \approx 0.973 G_{0}$, which is reasonably close to the exact value of $G_{0}$. The small deviation may be due to the minor uncertainty in the fitting and extrapolation procedures.

\section{Conductance of two-level Kondo QDs}

At the same temperature, the HEOM computation for a two-level QD is much more expensive than that for a singlelevel QD. The reason is mainly twofold: (i) the Fock space of a two-level QD is four times larger than that of a single-level QD, and hence, a much larger memory is needed; (ii) usually, a

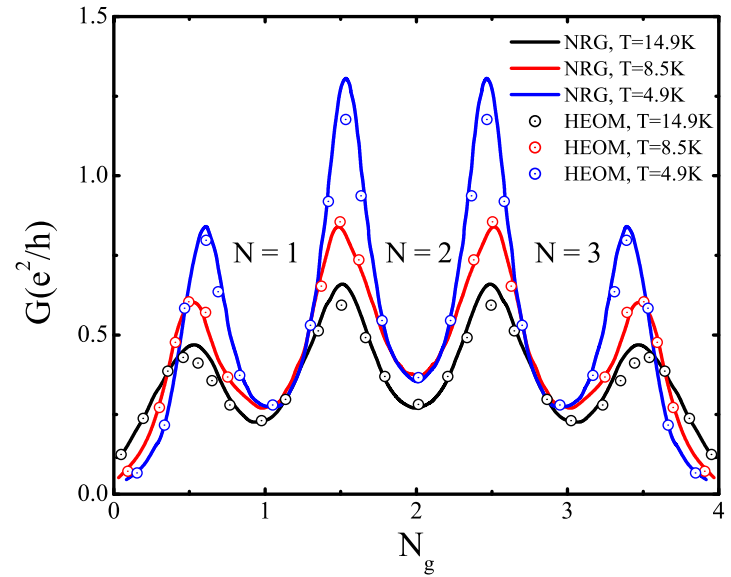

FIG. 15. (Color online) Comparison between HEOM (scattered symbols) and NRG (lines) calculated zero-bias conductance of a two-level QD. The NRG data are extracted from Fig. 1(a) of Ref. [73], which uses a constant hybridization function; the HEOM approach adopts a Lorentzian form of a hybridization function with a finite bandwidth $W=100 \Delta$. The dimensionless gate voltage $N_{g} \equiv \frac{1}{2}-$ $\epsilon_{1} / U$. The QD parameters are $\delta \epsilon=0$ and $U=20 \Delta$.

higher truncation level $L$ is required to accurately characterize the interlevel electron interactions. Therefore, with the limited computational resources at our disposal, it is very difficult to obtain the conductance of two-level QDs at very low $T$ and to examine the Kondo scaling behavior. Instead, we will compare the HEOM-calculated conductance with the NRG data in the literature at finite temperatures.

Anders et al. calculated the zero-bias conductance of QDs made from semiconducting carbon nanotubes with the NRG method [73]. A two-level Anderson model is adopted with $\delta \epsilon=0$. We calculate the conductance of the same QD with the HEOM approach, and the results are presented in Fig. 15. The calculation results exhibit both S-Kondo and O-Kondo features, as $G$ increases with decreasing $T$ in the $N=1,2,3$ valleys.

As shown in Fig. 15, the HEOM and NRG results agree well with each other in the whole range of gate voltage $\epsilon_{1}$. The remaining minor deviations (mainly in the conductance peak beside the $N=2$ valley) may be due to the different forms of hybridization functions adopted in the HEOM and NRG calculations; see the caption of Fig. 15 for details. We therefore have verified that the HEOM approach is capable of giving quantitatively accurate electronic properties for the two-level QDs.
[1] Y. Dubi and M. Di Ventra, Rev. Mod. Phys. 83, 131 (2011).

[2] G. G. Yadav, J. A. Susoreny, G. Q. Zhang, H. R. Yang, and Y. Wu, Nanoscale 3, 3555 (2011).

[3] M. G. Kanatzidis, Chem. Mater. 22, 648 (2010).

[4] M. G. Vavilov and A. D. Stone, Phys. Rev. B 72, 205107 (2005).

[5] A. Shakouri, Annu. Rev. Mater. Res. 41, 399 (2011).

[6] J. P. Small, K. M. Perez, and P. Kim, Phys. Rev. Lett. 91, 256801 (2003).
[7] S. F. Godijn, S. Möller, H. Buhmann, L. W. Molenkamp, and S. A. van Langen, Phys. Rev. Lett. 82, 2927 (1999).

[8] C. Yu, L. Shi, Z. Yao, D. Li, and A. Majumdar, Nano Lett. 5, 1842 (2005).

[9] R. Scheibner, H. Buhmann, D. Reuter, M. N. Kiselev, and L. W. Molenkamp, Phys. Rev. Lett. 95, 176602 (2005).

[10] R. Scheibner, E. G. Novik, T. Borzenko, M. König, D. Reuter, A. D. Wieck, H. Buhmann, and L. W. Molenkamp, Phys. Rev. B 75, 041301(R) (2007). 
[11] R. Scheibner, M. König, D. Reuter, A. D. Wieck, C. Gould, H. Buhmann, and L. W. Molenkamp, New J. Phys. 10, 083016 (2008).

[12] A. I. Hochbaum, R. Chen, R. D. Delgado, W. Liang, E. C. Garnett, M. Najarian, A. Majumdar, and P. Yang, Nature (London) 451, 163 (2008).

[13] S. Goswami, C. Siegert, M. Baenninger, M. Pepper, I. Farrer, D. A. Ritchie, and A. Ghosh, Phys. Rev. Lett. 103, 026602 (2009).

[14] A. A. Balandin, Nat. Mater. 10, 569 (2011).

[15] C. M. Jaworski, R. C. Myers, E. Johnston-Halperin, and J. P. Heremans, Nature (London) 487, 210 (2012).

[16] P. Reddy, S. Jang, R. Segalman, and A. Majumdar, Science 315, 1568 (2007).

[17] T. Naydenova, P. Dürrenfeld, K. Tavakoli, N. Pégard, L. Ebel, K. Pappert, K. Brunner, C. Gould, and L. W. Molenkamp, Phys. Rev. Lett. 107, 197201 (2011).

[18] N. Liebing, S. Serrano-Guisan, K. Rott, G. Reiss, J. Langer, B. Ocker, and H. W. Schumacher, Phys. Rev. Lett. 107, 177201 (2011).

[19] C. W. J. Beenakker and A. A. M. Staring, Phys. Rev. B 46, 9667 (1992).

[20] A. M. Lunde, K. Flensberg, and L. I. Glazman, Phys. Rev. Lett. 97, 256802 (2006).

[21] Y. Dubi and M. Di Ventra, Phys. Rev. B 79, 081302 (2009).

[22] T. A. Costi and V. Zlatić, Phys. Rev. B 81, 235127 (2010).

[23] J. Liu, Q.-F. Sun, and X. C. Xie, Phys. Rev. B 81, 245323 (2010).

[24] T. Rejec, R. Žitko, J. Mravlje, and A. Ramšak, Phys. Rev. B 85, 085117 (2012).

[25] E. Muñoz, C. J. Bolech, and S. Kirchner, Phys. Rev. Lett. 110, 016601 (2013).

[26] J. Azema, A.-M. Daré, S. Schäfer, and P. Lombardo, Phys. Rev. B 86, 075303 (2012).

[27] R.-Q. Wang, L. Sheng, R. Shen, B. Wang, and D. Y. Xing, Phys. Rev. Lett 105, 057202 (2010).

[28] V. Zlatić and J. K. Freericks, Phys. Rev. Lett. 109, 266601 (2012).

[29] D. Sánchez and R. López, Phys. Rev. Lett. 110, 026804 (2013).

[30] A. Ozaeta, P. Virtanen, F. S. Bergeret, and T. T. Heikkilä, Phys. Rev. Lett. 112, 057001 (2014).

[31] P. Mani, N. Nakpathomkun, E. A. Hoffmann, and H. Linke, Nano Lett. 11, 4679 (2011).

[32] M. Ciorga, A. S. Sachrajda, P. Hawrylak, C. Gould, P. Zawadzki, S. Jullian, Y. Feng, and Z. Wasilewski, Phys. Rev. B 61, R16315 (2000).

[33] T. Inoshita, A. Shimizu, Y. Kuramoto, and H. Sakaki, Phys. Rev. B 48, 14725 (1993).

[34] P. Jarillo-Herrero, J. Kong, H. S. J. van der Zant, C. Dekker, L. P. Kouwenhoven, and S. De Franceschi, Nature (London) 434, 484 (2005)

[35] K. Yamada, K. Yosida, and K. Hanzawa, Prog. Theor. Phys. 71, 450 (1984).

[36] R. Sakano and N. Kawakami, Phys. Rev. B 73, 155332 (2006).

[37] R. Sakano, T. Kita, and N. Kawakami, J. Phys. Soc. Jpn. 76, 074709 (2007).

[38] K. G. Wilson, Rev. Mod. Phys. 47, 773 (1975).

[39] R. Bulla, T. A. Costi, and T. Pruschke, Rev. Mod. Phys. 80, 395 (2008).

[40] R. Žitko and J. Bonča, Phys. Rev. B 74, 045312 (2006).

[41] R. Žitko and J. Bonča, Phys. Rev. B 76, 241305(R) (2007).
[42] J. E. Hirsch and R. M. Fye, Phys. Rev. Lett. 56, 2521 (1986).

[43] E. Gull, A. J. Millis, A. I. Lichtenstein, A. N. Rubtsov, M. Troyer, and P. Werner, Rev. Mod. Phys. 83, 349 (2011).

[44] M. Caffarel and W. Krauth, Phys. Rev. Lett. 72, 1545 (1994).

[45] E. Dagotto, Rev. Mod. Phys. 66, 763 (1994).

[46] Y. Meir, N. S. Wingreen, and P. A. Lee, Phys. Rev. Lett. 70, 2601 (1993).

[47] N. S. Wingreen and Y. Meir, Phys. Rev. B 49, 11040 (1994).

[48] J. S. Jin, X. Zheng, and Y. J. Yan, J. Chem. Phys. 128, 234703 (2008).

[49] X. Zheng, J. S. Jin, and Y. J. Yan, New J. Phys. 10, 093016 (2008).

[50] X. Zheng, J. S. Jin, S. Welack, M. Luo, and Y. J. Yan, J. Chem. Phys. 130, 164708 (2009).

[51] Z. H. Li, N. H. Tong, X. Zheng, D. Hou, J. H. Wei, J. Hu, and Y. J. Yan, Phys. Rev. Lett. 109, 266403 (2012).

[52] S. Wang, X. Zheng, J. S. Jin, and Y. J. Yan, Phys. Rev. B 88, 035129 (2013).

[53] X. Zheng, Y. J. Yan, and M. Di Ventra, Phys. Rev. Lett. 111, 086601 (2013).

[54] D. Hou, R. Wang, X. Zheng, N. H. Tong, J. H. Wei, and Y. J. Yan, Phys. Rev. B 90, 045141 (2014).

[55] S. M. Cronenwett, T. H. Oosterkamp, and L. P. Kouwenhoven, Science 281, 540 (1998).

[56] R. P. Feynman and F. L. Vernon, Jr., Ann. Phys. (N.Y.) 24, 118 (1963).

[57] X. Zheng, J. S. Jin, and Y. J. Yan, J. Chem. Phys. 129, 184112 (2008).

[58] Y. Wang, X. Zheng, B. Li, and J. Yang, J. Chem. Phys. 141, 084713 (2014).

[59] X. Zheng, R. Xu, J. Xu, J. Jin, J. Hu, and Y. Yan, Prog. Chem. 24, 1129 (2012).

[60] J. M. Ziman, Electrons and Phonons (Oxford University Press, Oxford, 1960).

[61] M. Wierzbicki and R. Swirkowicz, Phys. Rev. B 82, 165334 (2010).

[62] M. Turek and K. A. Matveev, Phys. Rev. B 65, 115332 (2002).

[63] B. Dong and X. L. Lei, J. Phys.: Condens. Matter 14, 11747 (2002).

[64] M. Cutler and N. F. Mott, Phys. Rev. 181, 1336 (1969).

[65] D. Goldhaber-Gordon, H. Shtrikman, D. Mahalu, D. AbuschMagder, U. Meirav, and M. A. Kastner, Nature (London) 391, 156 (1998).

[66] A. C. Hewson, The Kondo Problem to Heavy Fermions (Cambridge University Press, Cambridge, 1993).

[67] T. Pohjola, J. König, M. Salomaa, J. Schmid, H. Schoeller, and G. Schön, Europhys. Lett. 40, 189 (1997).

[68] D. C. Langreth, Phys. Rev. 150, 516 (1966).

[69] J. Martinek, M. Sindel, L. Borda, J. Barnaś, J. König, G. Schön, and J. von Delft, Phys. Rev. Lett. 91, 247202 (2003).

[70] D. Goldhaber-Gordon, J. Göres, M. A. Kastner, H. Shtrikman, D. Mahalu, and U. Meirav, Phys. Rev. Lett. 81, 5225 (1998).

[71] M. Grobis, I. G. Rau, R. M. Potok, H. Shtrikman, and D. Goldhaber-Gordon, Phys. Rev. Lett. 100, 246601 (2008).

[72] T. A. Costi, A. C. Hewson, and V. Zlatić, J. Phys.: Condens. Matter 6, 2519 (1994).

[73] F. B. Anders, D. E. Logan, M. R. Galpin, and G. Finkelstein, Phys. Rev. Lett. 100, 086809 (2008). 